\title{
The timing for initiating estrogen stimulation in artificial cycle for frozen-thawed embryo transfer can be flexible
}

Ying Ying ${ }^{\dagger} \mathbb{D}$, Yixuan $\mathrm{Wu}^{\dagger}$, Shuang Liu, Qing Huang ${ }^{*}$ and Haiying Liu*

\begin{abstract}
Background: There remains a lack of evidence to demonstrate whether the initiation time of estrogen stimulation is flexible in the proliferative endometrial phase during the artificial cycle for frozen-thawed embryo transfer (AC-FET).

Methods: FET records were retrospectively reviewed from a large university-affiliated reproductive medicine center. Only the patients who were undergoing their first embryo transfer with a single blastocyst in the AC-FET cycles were included: thereby 660 cycles were recruited, and the patients were grouped according to their day of estrogen usage initiation as early initiation group (estrogen stimulation initiated during days $2-5$ of menses, $n=128$ ) and the late initiation group (estrogen stimulation initiated on or after the 6 th day of menses, $n=532$ ). The primary outcome was the ongoing pregnancy rates (OPR).

Results: The rates of biochemical and clinical pregnancies were significantly higher in the late initiation group relative to those in the early initiation group, however, no significant differences were noted between the two groups for OPR. Furthermore, after adjusting for the results of the potential confounders, no impact was observed in the initiation time of estrogen stimulation on the OPR.
\end{abstract}

Conclusions: This study provides evidence that initiating the estrogen stimulation on after days 2-5 of menses do not exert adverse effects on the OPR in AC-FETs. Thus, AC-FET can be scheduled in a flexible manner without compromising on the pregnancy outcomes.

\section{Plain Language summary}

Despite the continuous efforts invested in exploring and optimizing therapeutic regimens to improve the success rate of frozen-thawed embryo transfer (FET), we found that there are little evidence to demonstrate whether the initiation time of estrogen stimulation is flexible in the proliferative endometrial phase during the artificial cycle for FET (ACFET). Thus, we retrospectively reviewed the FET records from a large university-affiliated reproductive medicine center to explore whether the relatively late start of endometrial stimulation for FET influences the pregnancy outcome. Results provided evidences that initiating the estrogen stimulation on after days $2-5$ of menses do not exert adverse

\footnotetext{
*Correspondence: kathyhuangqing@163.com; liuhaiying0606@163.com

†Ying Ying and Yixuan Wu contributed equally to this work

Department of Obstetrics and Gynecology, Center for Reproductive

Medicine, Key Laboratory for Major Obstetric Diseases of Guangdong

Province, The Third Affiliated Hospital of Guangzhou Medical University,

63 Duobao Road, Liwan District, Guangzhou, China
}

(c) The Author(s) 2021. Open Access This article is licensed under a Creative Commons Attribution 4.0 International License, which permits use, sharing, adaptation, distribution and reproduction in any medium or format, as long as you give appropriate credit to the original author(s) and the source, provide a link to the Creative Commons licence, and indicate if changes were made. The images or other third party material in this article are included in the article's Creative Commons licence, unless indicated otherwise in a credit line to the material. If material is not included in the article's Creative Commons licence and your intended use is not permitted by statutory regulation or exceeds the permitted use, you will need to obtain permission directly from the copyright holder. To view a copy of this licence, visit http://creativecommons.org/licenses/by/4.0/. The Creative Commons Public Domain Dedication waiver (http://creativeco mmons.org/publicdomain/zero/1.0/) applies to the data made available in this article, unless otherwise stated in a credit line to the data. 
effects on the ongoing pregnancy rates in AC-FETs. Therefore, FET can be scheduled in a flexible manner, according to the ovulatory and endometrial statuses and patient and/or clinic preference, without compromised clinical outcomes.

Keywords: Estrogen stimulation, Artificial cycle, Frozen-thawed embryo transfer

\section{Background}

Of late, the universal use of frozen-thawed embryo transfer (FET) in clinical practice has resulted in ideal pregnancy outcomes. Despite the dramatic rise in FET cycles over the recent years, there is insufficient evidence to recommend one protocol for endometrial preparation over another [1,2], and customized approaches are needed [3]. Artificial cycle FET (AC-FET) prepares the endometrium by the administration of exogenous estrogen and progesterone. The former promotes endometrial proliferation and induces progesterone receptors, while the latter alters the physiological state of the endometrium from the proliferation phase to the secretion phase. The series of hormonal changes make the endometrium receptive $[4,5]$. AC-FET mimics this physiological process.

To continuously improve the success rate of FET, several studies have attempted to optimize the therapeutic regimen by ascertaining the best doses and routes of administration for both estrogen and progesterone. Furthermore, efforts have been made to determine the optimum duration of estrogen administration prior to initiating progesterone administration. However, in clinical practice, different centers and even different doctors in the same center may show variations in the treatment protocols based on their individual concepts, experiences, and habits. For example, we know that when the pituitary is not suppressed using a gonadotropin releasing hormone agonist (GnRH-a), it is very important to start estrogen treatment in the early follicular phase (on day 1 or day 2), especially for those who ovulate regularly, because starting estrogen treatment after day 3 of the cycle might lead to an increased incidence of luteinizing hormone (LH) surge and the resultant luteinization of the endometrium [6]. However, we often encounter patients who come for consultation on the 6th day of their menstrual cycle or later and request endometrial preparation for FET. In general, such patients are those who had just completed oocyte retrieval and embryo cryopreservation, and they were instructed to consult the doctor on 3-5 days after the first menstruation is over, because they had to complete the leucorrhea examination before FET. If transvaginal ultrasound indicates no dominant follicle development in the bilateral ovaries, endometrial thickness is $<7 \mathrm{~mm}$, and serum sex hormones are at basal levels, in addition to the patients' time cost and personal willingness, the physician usually agrees to initiate the endometrial preparation program of AC-FET.
Therefore, we wished to ascertain whether the relatively late start of endometrial stimulation for FET influences the pregnancy outcome. Owing to the lack of a uniform standard, we defined "the late initiation of estrogen stimulation" as the onset of estrogen stimulation on or after the 6th day of natural menses or withdrawal bleeding. Thus, the present study was designed to assess whether the initiation time of estrogen stimulation in AC-FET cycles influences the pregnancy outcomes in patients undergoing their first cycle of vitrified-warmed, single blastocyst transfer.

\section{Methods \\ Study design}

This retrospective study was conducted at the Reproductive Medicine Center of The Third Affiliated Hospital of Guangzhou Medical University from January to December 2019. All FETs performed at our center during this period were reviewed for their potential inclusion in the study. Only patients who underwent their first embryo transfer after autologous in vitro fertilization (IVF) and single blastocyst thawing and transfer were included in this analysis. We identified AC-FETs without GnRHa pituitary downregulation and included the records of women $<45$ years of age at the time of FET. Since all patients underwent their first embryo transfer after IVF, all embryos were warmed and transferred within a year of vitrification. We excluded patients with preimplantation genetic testing (PGT) cycles, those with peak endometrial thickness of $<6 \mathrm{~mm}$, those with embryos derived from vitrified oocytes, and those with incomplete records. The study protocol was approved by the Ethical Committee of The Third Affiliated Hospital, Guangzhou Medical University.

\section{Frozen embryo transfer protocol}

Controlled ovarian stimulation, trigger injection, oocyte retrieval, embryo culture, embryo transfer, and cryopreservation were conducted according to the standard protocols. Fertilization was achieved via conventional IVF or intracytoplasmic sperm injection depending on the semen parameters and the history of prior IVF outcomes. Fresh embryo transfers were conducted either on Day 3 or Day 5 based on the embryo quality, number, and clinical indications. Similarly, the embryos were vitrified either in the cleavage or the blastocyst stage, depending on their quality, number, and clinical indications. 
FET was performed through a programmed or natural cycle as per the doctor's experience and the patient's specific situation and requirements. Only those women undergoing AC-FET without $\mathrm{GnRH}$-a downregulation were included in this study. On days $2-5$ of spontaneous menses or withdrawal bleeding, the patients underwent a baseline transvaginal ultrasound (TVUS) as well as an assessment of serum luteinizing hormone, estrogen, and progesterone to confirm that they were in the early proliferative phase of their menstrual cycle. In patients reporting late for various reasons as mentioned earlier, these investigations were performed on Day 6 of their menstrual cycle or beyond. Therapy was initiated with oral estrogen (estradiol valerate, Progynova, Bayer), $3 \mathrm{mg}$ twice daily. TVUS was performed within 7-10 days of initiating estrogen supplementation to assess the recipients' endometrium before adjusting the estrogen doses. Serum progesterone was measured at each visit to rule out premature ovulation before the initiation of progesterone supplementation. After at least 7 days of initiating estrogen administration, vaginal micronized progesterone supplementation ( $200 \mathrm{mg}$, three times per day) was started if the endometrial thickness was $>7 \mathrm{~mm}$. The transfer of frozen-thawed blastocysts was performed after 5 days of progesterone supplementation.

\section{Embryo grading}

The blastocysts were graded according to the following three morphological parameters: inner cell mass (ICM), trophectoderm, and the degree of expansion with a hatching stage [7]. At our center, blastocysts of grade $\geq 4 \mathrm{BB}$ were defined as high-quality blastocysts. Vitrification of the blastocysts was performed on days 5 or 6 based on the development of each embryo.

\section{Main outcome measures and statistical analyses}

The primary outcome measure was ongoing pregnancy, which was defined as the visualization of fetal cardiac activity on TVUS at $\geq 12$ weeks of gestation. The secondary outcomes included biochemical pregnancy (defined as cases with increased serum human chorionic gonadotropin levels 14 days after embryo transfer) and clinical pregnancy (confirmed by ultrasonographic visualization of a gestational sac 4-5 weeks after embryo transfer).

The continuous data were expressed as median (lower and upper quartiles), and the categorical data were expressed as counts (percentages). The parameters of the different groups were initially assessed using MannWhitney U-test for continuous variables and Chi-square test for categorical variables. Odds ratios (OR) with 95\% confidence intervals (CI) were calculated and adjusted for patient's age, body mass index (BMI), duration of estrogen usage, peak endometrial thickness, optimal embryos transferred, and initial day of estrogen administration using both univariable and multivariable logistic regression. All tests were two-sided and were performed using IBM Statistical Package for the Social Sciences software package, version 20. $\mathrm{p}<0.05$ was considered statistically significant.

\section{Results}

During the study period, a total of 6374 FET cycles were performed at our center. Among these, 660 cycles of ACFETs involving first embryo transfer (single-blastocyst transfer) were included in the present analysis. (Fig. 1).

Patients were divided into two groups according to the initial day of estrogen usage, as follows: early initiation group (estrogen stimulation started from days 2-5 of spontaneous menstruation or withdrawal bleeding, $\mathrm{n}=128$ ) and late initiation group (estrogen stimulation commenced on or after the 6th day of spontaneous menstruation or withdrawal bleeding, $\mathrm{n}=532$ ). The demographic characteristics and the FET parameters of the patients are summarized in Table 1. No significant differences were noted between the two groups in terms of the patients' age, BMI, infertility type and duration, infertility factor, and type of menstruation. However, the duration of estrogen usage was significantly longer

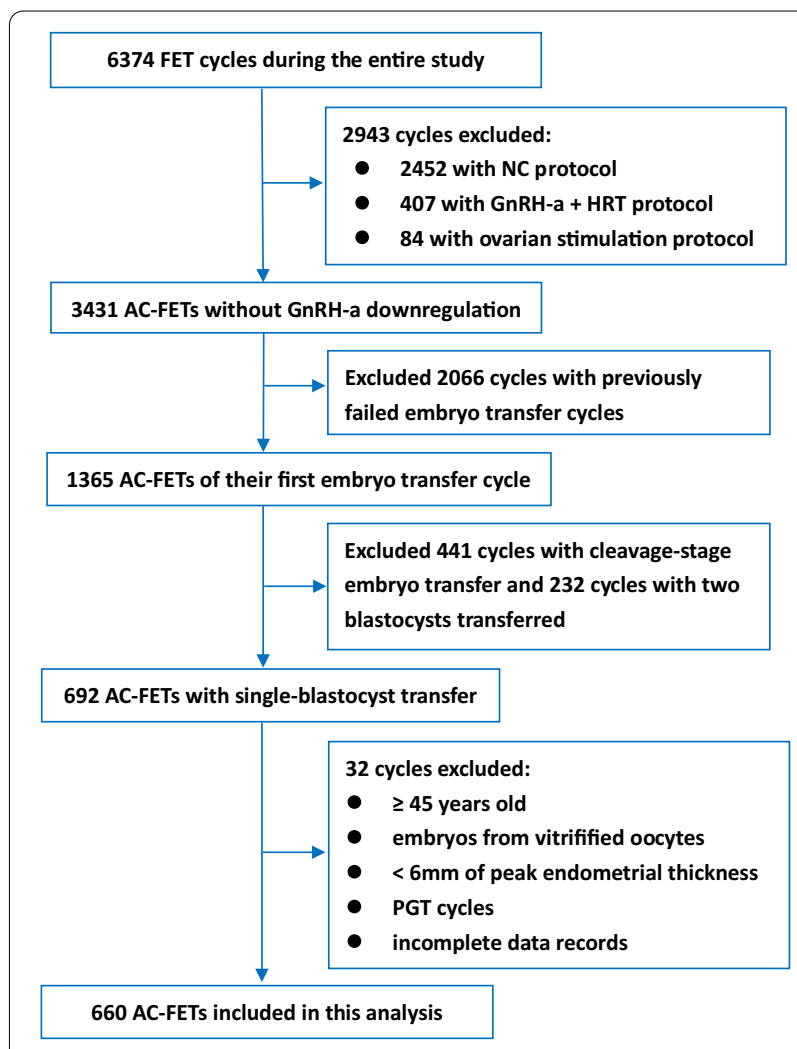

Fig. 1 Flowchart depicting the eligibility criteria for patient inclusion 
Table 1 Demographic characteristics and FET parameters of the study population according to the initial day of estrogen usage

\begin{tabular}{|c|c|c|c|}
\hline Parameters & Early initiation group $(n=128)$ & Late initiation group $(n=532)$ & $p$ value \\
\hline Age, years & $30(28,34)$ & $30(27,32)$ & 0.063 \\
\hline $\mathrm{BMl}, \mathrm{kg} / \mathrm{m}^{2}$ & $21.8(20.1,24.6)$ & $22.0(20.0,24.5)$ & 0.830 \\
\hline Infertility type, n (\%) & & & 0.486 \\
\hline Primary & $77(60.2)$ & $302(56.8)$ & \\
\hline Secondary & $51(39.8)$ & $230(43.2)$ & \\
\hline Infertility duration, years & $3(2,5)$ & $4(2,5)$ & 0.228 \\
\hline Infertility factor, n (\%) & & & 0.061 \\
\hline Tubal factor & $69(53.9)$ & $219(41.2)$ & \\
\hline Male factor & $19(14.8)$ & $93(17.5)$ & \\
\hline Mixed male and female factor & $33(25.8)$ & $191(35.9)$ & \\
\hline Unexplained & $7(5.5)$ & $29(5.5)$ & \\
\hline Menstruation, n (\%) & & & 0.993 \\
\hline Regular & $69(53.9)$ & $287(53.9)$ & \\
\hline Irregular & $59(46.1)$ & $245(46.1)$ & \\
\hline Duration of estrogen usage, days & $14(11,16)$ & $11(9,14)$ & $<0.001$ \\
\hline $\begin{array}{l}\text { Endometrial thickness at initial day of estrogen usage, } \\
\mathrm{mm}\end{array}$ & $5.0(4.1,5.8)$ & $5.9(4.0,5.7)$ & 0.469 \\
\hline Peak endometrial thickness, $\mathrm{mm}$ & $8.0(7.4,9.5)$ & $8.4(7.8,9.5)$ & 0.037 \\
\hline Optimal embryos transferred, n (\%) & $107(83.6)$ & $465(87.4)$ & 0.255 \\
\hline Biochemical pregnancy, n (\%) & $70(54.7)$ & $362(68.0)$ & 0.004 \\
\hline Clinical pregnancy, n (\%) & $66(51.6)$ & $342(64.3)$ & 0.008 \\
\hline Ongoing pregnancy, n (\%) & $60(46.9)$ & $290(54.5)$ & 0.120 \\
\hline
\end{tabular}

The continuous data were expressed as median (lower and upper quartiles)

in the early initiation group (14 days $[11,16])$ than in the late initiation group (11 days $[9,14])$. Besides, the peak endometrial thickness was significantly thicker in the late initiation group (8.4 $\mathrm{mm}[7.8,9.5])$ when compared with the early initiation group $(8.0 \mathrm{~mm}[7.4,9.5])$. Nonetheless, no significant differences were noted between the two groups in the endometrial thickness on the 1st day of estrogen usage and the percentages of optimal embryos transferred. The rates of biochemical and clinical pregnancies were significantly higher in the late initiation group than in the early initiation group $(68.0 \%$ versus $54.7 \%$ and $64.3 \%$ versus $51.6 \%$, respectively). However, no significant differences were observed between the two groups in the ongoing pregnancy rate.

Moreover, patients were divided into two groups according to the presence or absence of ongoing pregnancy, as follows: ongoing pregnancy group $(\mathrm{n}=350)$ and no ongoing pregnancy group $(n=310)$. The demographic characteristics and FET parameters of the patients are summarized in Table 2.

The patients in the ongoing pregnancy group were significantly younger than those in the no ongoing pregnancy group. Nevertheless, no significant differences were observed between the two groups in terms of BMI, infertility type and duration, infertility factor, and type of menstruation. Furthermore, the initial day and duration of estrogen usage, endometrial thickness on the 1st day of estrogen administration, and peak endometrial thickness were not significantly different between the two groups. The percentages of optimal embryos transferred were significantly higher in the ongoing pregnancy group than in the no ongoing pregnancy group $(91.7 \%$ versus $81.0 \%$, $\mathrm{p}<0.001$ ).

In the multivariable logistic regression model (Table 3), after adjusting the results for the potential confounders, including women's age, BMI, initial day of estrogen usage, duration of estrogen usage, peak endometrial thickness, and optimal embryos transferred, only the women's age (OR 0.942, 95\% CI 0.904-0.982, $\mathrm{p}=0.005$ ) and morphologically optimal embryos transferred (OR 2.321, 95\% CI $1.429-3.770, \mathrm{p}=0.001$ ) were inferred to be important independent prognostic factors for confirming an ongoing pregnancy. Thus, the results suggested that the initial time of estrogen stimulation should not be considered as a prognostic factor for ongoing pregnancy.

\section{Discussion}

To date, many studies have explored ways to optimize AC-FET in various aspects to make the endometrium receptive, including the selection of the best doses and 
Table 2 Demographic characteristics and FET parameters of the study population according to ongoing pregnancy

\begin{tabular}{|c|c|c|c|}
\hline Parameters & Ongoing pregnancy $(n=350)$ & No ongoing pregnancy $(n=310)$ & $p$ value \\
\hline Age, years & $29(27,32)$ & $30(28,34)$ & 0.001 \\
\hline $\mathrm{BMI}, \mathrm{kg} / \mathrm{m}^{2}$ & $21.8(20.1,24.0)$ & $22.2(20.0,25.0)$ & 0.113 \\
\hline Infertility type, n (\%) & & & 0.751 \\
\hline Primary & $203(58.0)$ & $176(56.8)$ & \\
\hline Secondary & $147(42.0)$ & $134(43.2)$ & \\
\hline Infertility duration, years & $3.5(2,5)$ & $3(2,5)$ & 0.549 \\
\hline Infertility factor, n (\%) & & & 0.349 \\
\hline Tubal factor & $164(46.9)$ & $124(40.0)$ & \\
\hline Male factor & $55(15.7)$ & $57(18.4)$ & \\
\hline Mixed male and female factor & $112(32.0)$ & $112(36.1)$ & \\
\hline Unexplained & $19(5.4)$ & $17(5.5)$ & \\
\hline Menstruation, n (\%) & & & 0.902 \\
\hline Regular & $188(53.7)$ & $168(54.2)$ & \\
\hline Irregular & $162(46.3)$ & $142(45.8)$ & \\
\hline Initial day of estrogen usage & $11(8,13)$ & $10(7,13)$ & 0.387 \\
\hline Duration of estrogen usage, days & $11(10,15)$ & $11(9,15)$ & 0.331 \\
\hline Endometrial thickness at initial day of estrogen, $\mathrm{mm}$ & $5.0(4.0,5.7)$ & $4.8(4.1,5.8)$ & 0.879 \\
\hline Peak endometrial thickness, mm & $8.5(7.8,9.6)$ & $8.2(7.6,9.4)$ & 0.076 \\
\hline Optimal embryos transferred, n (\%) & $321(91.7)$ & $251(81.0)$ & $<0.001$ \\
\hline
\end{tabular}

The continuous data were expressed as median (lower and upper quartiles)

Table 3 Univariable and multivariable logistic regression analysis of factors related to ongoing pregnancy

\begin{tabular}{|c|c|c|c|c|}
\hline \multirow[t]{2}{*}{ Variables } & \multicolumn{2}{|l|}{ Univariable analysis } & \multicolumn{2}{|c|}{ Multivariable analysis } \\
\hline & OR $(95 \% \mathrm{Cl})$ & pvalue & OR $(95 \% \mathrm{Cl})$ & pvalue \\
\hline Age & $0.928(0.892-0.966)$ & $<0.001$ & $0.942(0.904-0.982)$ & 0.005 \\
\hline BMI & $0.961(0.920-1.005)$ & 0.083 & $0.965(0.922-1.010)$ & 0.129 \\
\hline Initial day of estrogen usage & $1.016(0.978-1.054)$ & 0.420 & $1.021(0.981-1.063)$ & 0.308 \\
\hline Duration of estrogen usage & $1.018(0.979-1.059)$ & 0.371 & $1.037(0.992-1.084)$ & 0.109 \\
\hline Peak endometrial thickness & $1.081(0.969-1.205)$ & 0.162 & $1.115(0.991-1.255)$ & 0.070 \\
\hline Optimal embryos transferred & $2.602(1.619-4.180)$ & $<0.001$ & $2.321(1.429-3.770)$ & 0.001 \\
\hline
\end{tabular}

routes of administration for both estrogen and progesterone as well as the optimum duration of estrogen administration prior to initiating progesterone.

To the best of our knowledge, this is the first study to determine whether the initial time of estrogen stimulation influences the ongoing pregnancy in AC-FET. At the very beginning, downregulation of $\mathrm{GnRH}-\mathrm{a}$ was important before estrogen stimulation in AC-FET cycles. In the later stage, it turned out that the $\mathrm{LH}$ rise and endometrium luteinization rarely occurred without GnRH-a downregulation. However, if the pituitary is not suppressed using a GnRH-a, it is very important to start estrogen treatment in the early follicular phase (on Day 1 or Day 2), especially for those with normal ovulation. Therefore, estrogen stimulation is routinely started on days 2-5 of menstruation or withdrawal bleeding in our center. Nonetheless, many patients do start hormone treatment beyond this window owing to various reasons, and this happens quite often in our center. We did not find that this delay has an impact on the overall success rate.

Our data showed that patients with late estrogen initiation (on or after the 6th day of natural menses or withdrawal bleeding) shared similar ongoing pregnancy rates as those who initiated the treatment within the normal time range. Significant differences were however noted in the duration of estrogen usage and the peak endometrial thickness between the two groups. After adjusting the results for the potential confounders, including the above-mentioned parameters, it was evident the initial 
time of estrogen stimulation should not be viewed as an important independent prognostic factor for confirming ongoing pregnancy.

A duration of 10-14 days of estrogen administration is routinely utilized in artificial protocols; however, for those patients who do not respond within this time, the duration should be extended. Histological evaluations have revealed that 5 weeks of estrogen priming is ideal for obtaining optimal luteal phase endometrial histology after progesterone treatment [8]. Positive results have been reported after prolonged ( $4-5$ weeks) vaginal estrogen administration [9]. Therefore, there does not appear to be an upper limit for the duration of estrogen stimulation. However, one study has reported that prolonged unopposed estrogen administration beyond 40 days is associated with a high rate of breakthrough bleeding [10]. In our study, the longest duration of estrogen administration was 26 days, and no breakthrough bleeding was observed in the study population.

When the patients were divided into ongoing pregnancy and no ongoing pregnancy groups, no significant difference was found in the initial day of estrogen usage between the two groups. The patients were significantly younger and the percentages of optimal embryos transferred were significantly higher in the ongoing pregnancy group than in the other group. However, after adjusting the results for the potential confounders using multivariate logistic regression analysis, the results signified that the initial time of estrogen stimulation should not be considered as a prognostic factor for ongoing pregnancy.

The strength of our study lies in our inclusion of the impacts of many factors such as the women's age, previous failed embryo transfer cycles, embryo development stage, number of embryos transferred, and endometrial thicknesses. Despite our cautions, our study has certain limitations. The first one is related to the inevitable bias introduced by the retrospective nature of the study. Second, some baseline characteristics differed between the groups, after adjusting for the confounders, we still observed that the initial time of estrogen stimulation did not have an impact on the ongoing pregnancy rate. Finally, we did not analyze the live birth rates because of the non-availability of complete patients records. Despite these limitations, our study provides valuable data for the physicians employing the flexible artificial protocol.

\section{Conclusions}

This study provides evidence that initiating estrogen stimulation beyond the window of 2-5 days of menses or withdrawal bleeding does not adversely affect the ongoing pregnancy. Our findings imply that AC-FET can be scheduled in a flexible manner without compromising the pregnancy outcome. However, well-designed, prospective clinical trials are needed to further assess the effects of late initiation of estrogen administration in AC-FET.

\section{Abbreviations}

AC-FET: Artificial cycle for frozen-thawed embryo transfer; OPR: Ongoing pregnancy rates; GnRH-a: Gonadotropin releasing hormone agonist; LH: Luteinizing hormone; IVF: In vitro fertilization; PGT: Preimplantation genetic testing; TVUS: Transvaginal ultrasound.

\section{Acknowledgements}

Not applicable.

\section{Authors' contributions}

YY conceived the original idea, designed the study, and wrote the manuscript. YW and SL performed data analysis. QH and HL helped supervise the study and revised the paper. All authors read and approved the final manuscript.

\section{Funding}

This study was supported by the National Natural Science Foundation of China (No.81701518 and No.81801532).

\section{Availability of data and materials}

The datasets used and/or analyzed during the current study are available from the corresponding author on reasonable request.

\section{Declarations}

Ethics approval

Medical Ethics Committee of The Third Affiliated Hospital of Guangzhou Medical University approved the study (Approval No. [2021]-015).

\section{Consent to participate}

Not applicable.

\section{Consent for publication}

Not applicable.

\section{Competing interests}

The authors declare that they have no competing interests.

Received: 15 June 2021 Accepted: 20 August 2021

Published online: 09 September 2021

\section{References}

1. Glujovsky D, Pesce R, Sueldo C, Quinteiro Retamar AM, Hart RJ, Ciapponi A. Endometrial preparation for women undergoing embryo transfer with frozen embryos or embryos derived from donor oocytes. Cochrane Database Syst Rev. 2020;10:CD006359. https://doi.org/10.1002/14651858. CD006359.pub3.

2. Ghobara T, Gelbaya TA, Ayeleke RO. Cycle regimens for frozen-thawed embryo transfer. Cochrane Database Syst Rev. 2017;7(7):CD003414. https://doi.org/10.1002/14651858.CD003414.pub3.

3. Groenewoud ER, Cantineau AE, Kollen BJ, Macklon NS, Cohlen BJ. What is the optimal means of preparing the endometrium in frozen-thawed embryo transfer cycles? A systematic review and meta-analysis. Hum Reprod Update. 2013;19(5):458-70. https://doi.org/10.1093/humupd/ dmt030. Erratum in: Hum Reprod Update. 2017 Mar 1;23(2):255-61. (Epub 2013 Jul 2)

4. Lessey BA, Killam AP, Metzger DA, Haney AF, Greene GL, McCarty KS Jr. Immunohistochemical analysis of human uterine estrogen and progesterone receptors throughout the menstrual cycle. J Clin Endocrinol Metab. 1988;67(2):334-40. https://doi.org/10.1210/jcem-67-2-334.

5. Noyes RW, Hertig AT, Rock J. Dating the endometrial biopsy. Am J Obstet Gynecol. 1975;122(2):262-3. https://doi.org/10.1016/s0002-9378(16) 33500-1. 
6. Remohi J, Vidal A, Pellicer A. Oocyte donation in low responders to conventional ovarian stimulation for in vitro fertilization. Fertil Steril. 1993;59(6):1208-15. https://doi.org/10.1016/s0015-0282(16)55978-0.

7. Gardner DK, Lane M, Stevens J, Schlenker T, Schoolcraft WB. Blastocyst score affects implantation and pregnancy outcome: towards a single blastocyst transfer. Fertil Steril. 2000;73(6):1155-8. https://doi.org/10. 1016/s0015-0282(00)00518-5.

8. Navot D, Laufer N, Kopolovic J, Rabinowitz R, Birkenfeld A, Lewin A, Granat $M$, Margalioth EJ, Schenker JG. Artificially induced endometrial cycles and establishment of pregnancies in the absence of ovaries. N Engl J Med. 1986;314(13):806-11. https://doi.org/10.1056/NEJM198603273141302.

9. Tourgeman DE, Slater CC, Stanczyk FZ, Paulson RJ. Endocrine and clinical effects of micronized estradiol administered vaginally or orally. Fertil Steril. 2001;75(1):200-2. https://doi.org/10.1016/s0015-0282(00)01640-x.
10. Borini A, Dal Prato L, Bianchi L, Violini F, Cattoli M, Flamigni C. Effect of duration of estradiol replacement on the outcome of oocyte donation. J Assist Reprod Genet. 2001;18(4):185-90. https://doi.org/10.1023/a:10094 72416305.

\section{Publisher's Note}

Springer Nature remains neutral with regard to jurisdictional claims in published maps and institutional affiliations.
Ready to submit your research? Choose BMC and benefit from:

- fast, convenient online submission

- thorough peer review by experienced researchers in your field

- rapid publication on acceptance

- support for research data, including large and complex data types

- gold Open Access which fosters wider collaboration and increased citations

- maximum visibility for your research: over $100 \mathrm{M}$ website views per year

At BMC, research is always in progress.

Learn more biomedcentral.com/submissions 